\title{
An uncommon manifestation of paraneoplastic cerebellar degeneration in a patient with high grade urothelial, carcinoma with squamous differentiation: A case report and literature review
}

Yaofeng Zhu ${ }^{1 \dagger}$, Shouzhen Chen ${ }^{1 \dagger}$, Songyu Chen ${ }^{2}$, Jing Song ${ }^{3}$, Fan Chen ${ }^{1}$, Hu Guo ${ }^{1}$, Zhenhua Shang ${ }^{1}$, Yong Wang ${ }^{1}$, Changkuo Zhou ${ }^{1}$ and Benkang Shi ${ }^{*}$

\begin{abstract}
Background: Paraneoplastic neurological syndromes (PNS) are rare disorders associated with malignant tumours, which are triggered by autoimmune reactions. Paraneoplastic cerebellar degeneration (PCD) is the PNS type most commonly associated with ovarian and breast cancer. Two bladder cancers manifesting in PCD were previously reported. However, the cancers in these cases had poor outcomes.
\end{abstract}

Case presentation: Here, we present a 68-year old man with history of high-grade papillary urothelial carcinoma of the bladder. The patient suffered from persistent cerebellar ataxia accompanied by bladder cancer recurrence five months after transurethral resection of the bladder tumour (TURBt). Laboratory screening for the specific antibodies of paraneoplastic neurological syndromes revealed no positive results. Symptoms were not remitted after a 7-day-course of high-dose glucocorticoid therapy. To our surprise, the patient recovered fully after laparoscopic radical cystectomy. Postoperative pathology revealed that surgical specimens were urothelial carcinoma in situ (CIS) and squamous cell carcinoma of the bladder. The patient remained asymptomatic and there was no evidence of recurrence after the followup period of 11 months.

Conclusion: To our knowledge, this is the third report of PCD in a patient with bladder cancer. This case showed that tumour resection cured the PCD. To assist clinical evaluation and management, literature regarding basic PNS characteristics and bladder cancers was reviewed.

Keywords: Paraneoplastic cerebellar degeneration, Bladder cancer, High grade urothelial, Carcinoma, Squamous differentiation

\section{Background}

The bladder cancer is the 11th most common site of cancer diagnosis and the 14th leading cause of cancerrelated deaths in the world [1]. The 2004 WHO/ISUP system classifies papillary urothelial neoplasms into four types: papilloma, papillary urothelial neoplasm of low

\footnotetext{
* Correspondence: benkangshi@126.com

${ }^{\dagger}$ Equal contributors

'Department of Urology, Qilu Hospital of Shandong University, Wenhua Xi Road, Jinan, Shandong Province, People's Republic of China

Full list of author information is available at the end of the article
}

malignant potential, low-grade papillary urothelial carcinoma, and high-grade papillary urothelial carcinoma [2]. The high-grade papillary urothelial carcinoma was associated with the high recurrence rate. The main clinical treatment for non-muscle-invasive bladder cancer is transurethral resection of $\mathrm{Ta}$ and $\mathrm{T} 1$ bladder tumours [3]. However, carcinoma in situ (CIS) is a high-grade, flat, non-invasive urothelial form. This carcinoma is often multifocal and progresses to muscle-invasive disease. Therefore, endoscopic procedures alone are insufficient for CIS treatment. Either intravesical bacillus 
Calmette-Guérin (BCG) instillations or radical cystectomies (RC) are applied $[4,5]$. Several studies have revealed excellent outcomes after immediate RC for CIS [6].

Paraneoplastic neurological syndromes (PNS) are rare disorders associated with malignant tumours that are not directly caused by tumour invasion and metastasis. The autoimmune response is elicited by the ectopic expression of neural antigens in neoplastic tissues, which eventually attack the nervous system of PNS patients [7]. PNS incidence is estimated to be $0.5-1 \%$, varying by cancer type $[8,9]$. Paraneoplastic cerebellar degeneration (PCD) is the type of PNS most commonly associated with ovarian and breast cancer [9]. PCD is characterized by subacute cerebellar ataxia within 12 weeks and subsequent cerebellar atrophy [10]. Bladder cancers presenting with PCD have rarely been reported. Greenlee JE [11] and Tetsuka S [12] reported PCD and its causal antibody in bladder cancer patients. Here, we discuss a patient with urothelial CIS of the bladder who suffered from PCD. Unlike two previous cases, we found no specific antibody causing PCD. Literature concerning unusual cases are reviewed.

\section{Case presentation}

A 68-year-old man was admitted to the Qilu Hospital of Shandong University on November 17, 2014 for urinary irritation, dysuria and haematuria. The patient's condition was good, and weight loss, night sweats and recent fever did not present. Urinalysis showed red and white blood cell counts of $61.4 / \mu \mathrm{L}$ and $313.8 / \mu \mathrm{L}$, respectively. Cystoscopy showed scattered cauliflower-like neoplasms located at $11^{\circ}-1^{\circ}$ of the narrow bladder neck, with a maximum diameter of $1.5 \mathrm{~cm}$. A biopsy was then conducted and the pathological examination revealed that the neoplasms were high-grade papillary urothelial carcinoma of the bladder. CT scanning showed a thickened anterior bladder wall, without enlarged pelvic lymph nodes. Transurethral resection of the bladder tumour (TURBt) was conducted after a definitive diagnosis was made. Several villous tumours were found on the neck of the bladder during the operation. The postoperative pathological examination showed that the surgical specimens were high-grade papillary urothelial carcinoma accompanied by differentiated squamous cell carcinoma. This carcinoma also involved the prostate. Haematuria and dysuria symptoms disappeared after TURBt. Therefore, the patient's family refused further treatment and the patient was discharged.

The patient was referred to our hospital again on March 27, 2015 because of vomiting, progressive gait imbalance and Haematuria for the previous five days. Neurological examinations revealed ataxic gait and bilateral coarse nystagmus. Slight dysmetria was confirmed by finger-to-nose and heel-knee-shin testing. Haematuria led to the performance of a urinalysis showing red and white blood cell counts of $554 / \mu \mathrm{L}$ and $6748 / \mu \mathrm{L}$, respectively. Further cystoscopy revealed that bladder mucosa located at $11^{\circ}-1^{\circ}$ of the bladder neck suffered hyperaemia, edema, and erosion of the asperous surface. The biopsy showed a high-grade papillary urothelial carcinoma and squamous differentiation.

Further laboratory and radiographic evaluations were conducted to clarify the relationship between bladder cancer recurrence and emerging cerebellar ataxia. A brain MRI was performed to exclude brain metastases from bladder cancer (Fig. 1 c, d). The MRI revealed no obvious morphological change of the cerebellum (Fig. 1 $\mathrm{a}, \mathrm{b})$. The abdominal and pelvic CT scans revealed that the bladder wall was thickened, especially at the neck (Fig. 2). The above radiographic findings suggest little possibility exists that nervous symptoms were triggered by brain metastases from bladder cancer.

Further laboratory examinations were conducted to validate the cause of neurological symptoms. Nine common types of paraneoplastic antibodies were all negative in the serum and cerebrospinal fluid (CSF). Details of the paraneoplastic antibodies are shown in Table 1. The CSF examination revealed an elevated level of protein $(58.6 \mathrm{mg} / \mathrm{dl}$, normal: $15-45 \mathrm{mg} / \mathrm{dl})$, IgG $(39.5 \mathrm{mg} / \mathrm{L}$, normal $<34 \mathrm{mg} / \mathrm{L})$, and albumin $(391 \mathrm{mg} / \mathrm{L}$, normal < $350 \mathrm{mg} / \mathrm{L}$ ). Pandy's test of CSF was positive, while CSF cell counts were normal. Tumour markers showed that squamous cell cancer antigen (SCC) and non-small cell lung cancer antigen (CYFRA21-1) serum concentrations were elevated (SCC: $6 \mathrm{ng} / \mathrm{ml}$, normal: $0.1-0.3 \mathrm{ng} /$ $\mathrm{ml}$; CYFRA21-1: $3.5 \mathrm{ng} / \mathrm{ml}$, normal $<1.5 \mathrm{ng} / \mathrm{ml})$. The thyroglobulin serum concentration was low $(<0.04 \mathrm{ng} / \mathrm{ml}$, normal: $1.4-78 \mathrm{ng} / \mathrm{ml}$ ). The examination of rheumatic antigens revealed no positive results. The electroencephalogram revealed no epileptogenic activity.

Based on these findings, we suspected that the patient suffered from PCD. The patient initially received a 5-day course of methylprednisolone (500 mg intravenously daily) without significant clinical improvement. Considering the first postoperative pathological examination revealed the bladder cancer involved the prostate and recurred, the patient underwent laparoscopic radical cystectomy (LRC). The postoperative pathological examination revealed that the surgically removed bladder was urothelial CIS and well-differentiated squamous cell carcinoma (Fig. 3). This finding was in accordance with the changes in serum SCC and CYFRA21-1. The squamous cell carcinoma was dispersed in the bladder mucosa (Fig. 3a) and accounted for approximately $5 \%$ of all carcinomas. Neurological symptoms disappeared, and basic daily living activities were obviously improved, according to Barthel score (Table 2), after LRC. The patient remained asymptomatic and there was no evidence of recurrence after the followup period of 11 months. 

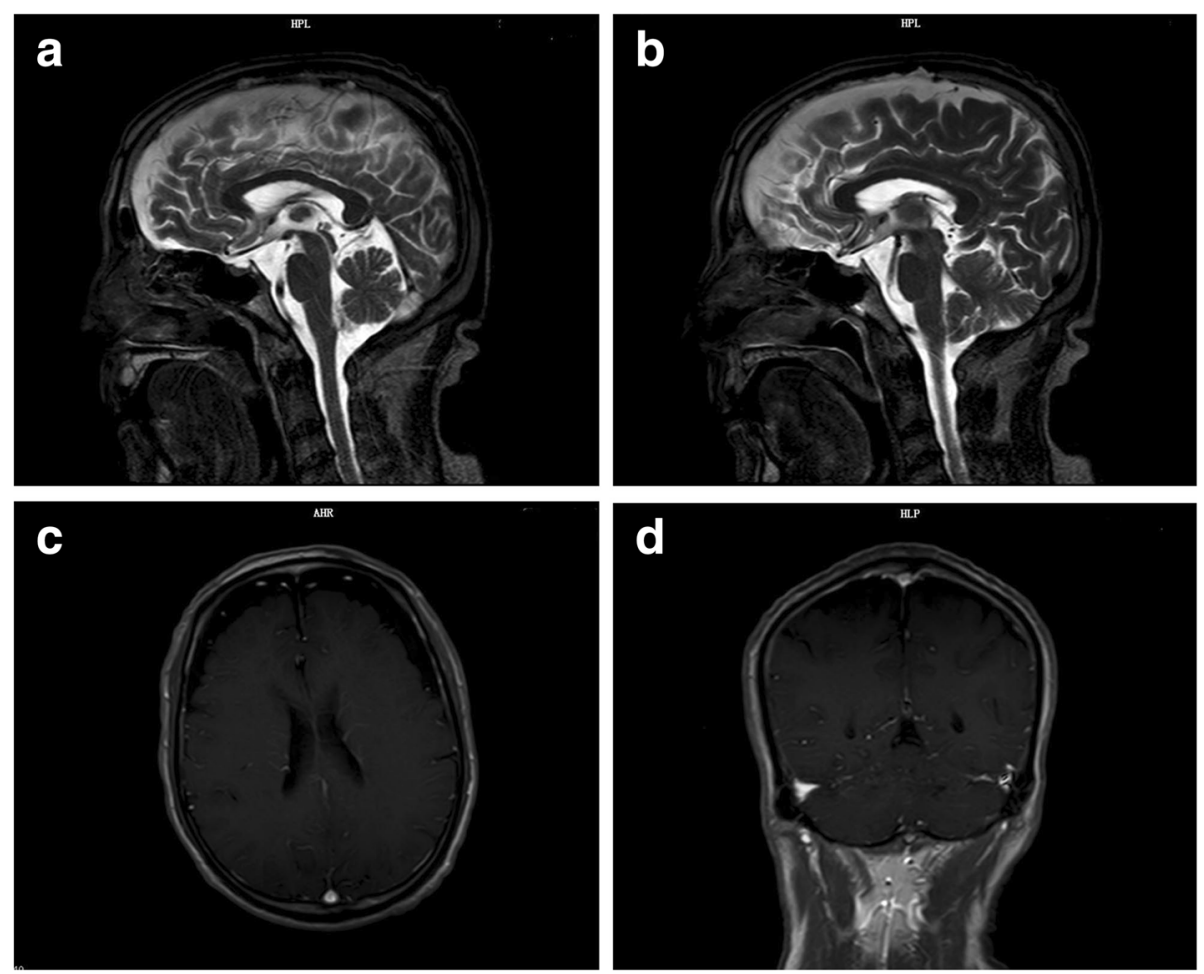

Fig. 1 Magnetic resonance imaging (MRI) of the brain. $\mathbf{a}$ and $\mathbf{b}$, the T2WI sagittal scan showed no obvious morphological change of the cerebellum; c and $\mathbf{d}$, the enhanced-scanning MRI revealed no sign of brain metastases from bladder cancer

\section{Discussion}

PNS was initially defined as the presence of cancer and the exclusion of other known causes of the neurological symptoms. Examples of such causes include metastasis, coagulopathy, infections, metabolic or nutritional disturbances, and treatment-induced neurotoxicity [10, 13]. The exact pathogenesis of most PNS is unknown. However, neuronal antigens expressed by cancers could activate the immune system. Moreover, many antineuronal antibodies generated by the activated immune system attack the nervous system [7]. Antineuronal antibodies can affect different nervous system regions, and multiple clinical manifestations can present in PNS. When the cerebellum is affected, the rapid development of severe cerebellar ataxia is caused by extensive Purkinje neurons loss, defined as PCD.

According to the PNS Euronetwork recommended diagnostic framework in 2004 [13], the following criteria are required to define PCD: (1) severe pancerebellar syndrome developing in less than 12 weeks; (2) no radiographic evidence of cerebellar atrophy other than that expected by the patient's age; and (3) the significant interference of symptoms caused by PCD with basic activities of daily living. Neurological syndromes developed in five days and the MR examnation excluded cerebellar atrophy in the present case. The Barthel activities of daily living Index was 35 after the onset of the disease, indicating a severe effect. All the characteristics in the
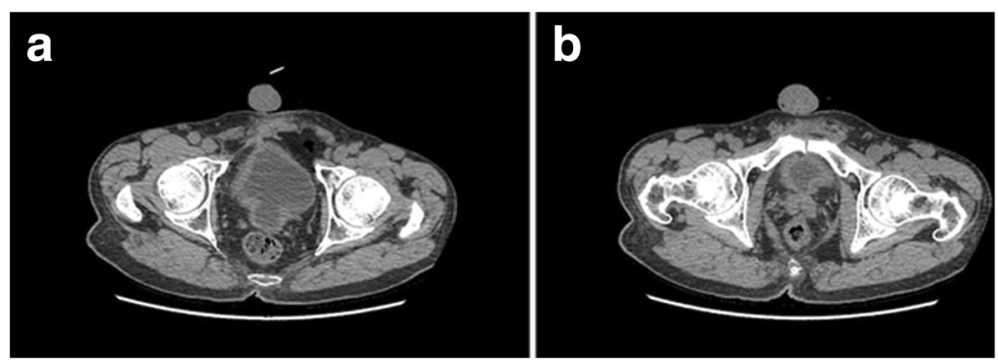

Fig. 2 Computed tomography $(\mathrm{CT})$ of the abdomen and pelvis. A, the bladder wall was thickened; B, the neck of bladder was thicker than the bladder wall 
Table 1 Examination Results of Paraneoplastic Antibodies

\begin{tabular}{ll}
\hline Type of antibodies: Ig G & \\
Antibody Description & Results \\
\hline Anti-Amphiphysin & $(-)$ \\
Anti-CV2.1 & $(-)$ \\
Anti-PNMA2/Ta & $(-)$ \\
Anti-Ri & $(-)$ \\
Anti-Yo & $(-)$ \\
Anti-Hu & $(-)$ \\
Anti-Recoverin & $(-)$ \\
Anti-SOX1 & $(-)$ \\
Anti-Titin & $(-)$ \\
\hline
\end{tabular}

$(-)=$ negative

present case met PCD diagnostic criteria. Other possible reasons for neurological syndromes were excluded. The neurological syndromes associated with bladder cancer could be clinically diagnosed as PCD.

PNS is commonly associated with ovarian cancer, breast cancer, SCLC, or Hodgkin's disease. Few reports have associated PNS with bladder cancer. We retrospectively reviewed English literature regarding PNS and bladder cancers. We used PubMed, Medscape and EMBASE to search for studies on this topic, yielding eight articles. Only two reported a relationship between PCD and bladder cancer. Therefore, this study is the third to describe the relationship between PCD and bladder cancer. Clinical and pathological features were summarized according to previous reports and the present case (Table 3).

The male to female ratio of 2 to 1 in the nine related cases is different from the bladder cancer incidence rate. The worldwide incidence rate is believed to be 8.9 / 100,000 and 2.2/100,000 for men and women, respectively. Therefore, the male to female ratio is more than 4 to 1 [1]. Sean et al. reported that the female to male ratio of 34 PNS patients seropositive for anti-Ri was 2 to
Table 2 Barthel Index of Activities of Daily Living Before and After LRC

\begin{tabular}{llllll}
\hline Activities & Independent & Needs help & Unable & Before LRC & After LRC \\
\hline Feeding & 10 & 5 & 0 & 5 & 10 \\
Transfer & 15 & 5 or 10 & 0 & 0 & 10 \\
Grooming & 5 & 0 & $/$ & 0 & 5 \\
Toilet use & 10 & 5 & 0 & 5 & 10 \\
Bathing & 5 & 0 & $/$ & 0 & 5 \\
Dressing & 10 & 5 & 0 & 5 & 10 \\
Mobility & 15 & 5 or 10 & 0 & 0 & 10 \\
Stairs & 10 & 5 & 0 & 0 & 5 \\
Bowels & 10 & 5 & 0 & 10 & 10 \\
Bladder & 10 & 5 & 0 & 10 & 10 \\
Total Score & 100 & & & 35 & 85 \\
\hline
\end{tabular}

1 [14]. British data showed a striking female preponderance of PNS, with a female to male ratio of 2.3 to 1 [15]. Therefore, the female patients with bladder cancer may be predisposed to PNS. This finding is the same as for PNS caused by other cancers.

PNS is caused by high-grade muscle-invasive bladder cancer in most cases. Blood supplies of the muscleinvasive bladder cancer are richer, facilitating the circulation of neuronal antigens expressed by high-grade cancers. In the present case, the patient with the urothelial CIS and squamous cell carcinoma of the bladder suffered from PCD. Matsumoto L. reported that CIS of the testis triggered severe hypokinesis as a paraneoplastic manifestation and detection of anti-Ma2 antibodies [16]. In this case, the bladder cancers comprised high-grade urothelial carcinoma and well-differentiated squamous cell carcinoma. However, methods of verifying which type of carcinoma causing PCD are limited. Gita reported that recurrent bladder cancers with squamous features caused paraneoplastic encephalomyelitis [17]. Numerous reports of other organs have also demonstrated that PNS was caused by squamous cell carcinoma [18, 19]. Therefore,

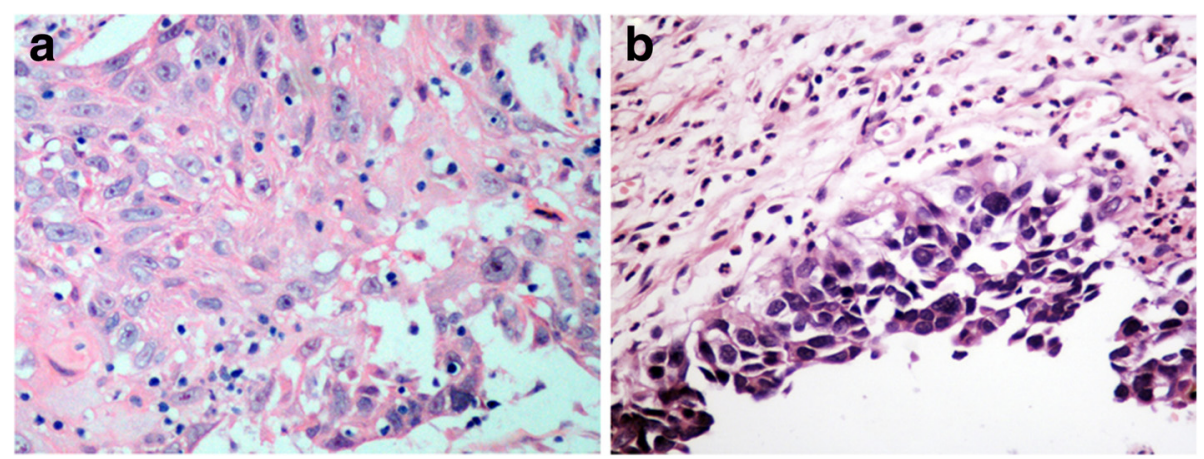

Fig. 3 HE staining of bladder cancer tissues. a, HE staining showed irregular squamous cell carcinoma dispersed in the bladder mucosa. b, HE staining showed the urothelial CIS 
Table 3 Clinical and Pathological Features in Nine Cases of Bladder Cancer with PNS

\begin{tabular}{|c|c|c|c|c|c|c|c|c|c|}
\hline Case No. & Author & Year & Age/Gender & Stage & Pathology & Clinical syndromes & Antibody & Treatment & Prognosis \\
\hline 1 & Gita et al.[17] & 2015 & $73 / F$ & $M^{a}$ & $\begin{array}{l}\text { Poorly differentiated carcinoma with } \\
\text { squamous features }\end{array}$ & PEM & Negtive & $\begin{array}{l}\text { Surgical resection and } \\
\text { immunosuppression }\end{array}$ & Partial improvement \\
\hline 2 & Syuichi et al.[12] & 2013 & $66 / M$ & N/A & Urothelial carcinoma & PCD & Anti-CKB & TURBT & No improvement \\
\hline 3 & Lukacs et al.[20] & 2012 & $76 / F$ & pTa & Urothelial carcinoma & PEM and SSN & Anti-Hu & TURBT & Partial improvement \\
\hline 4 & Forte et al.[21] & 2009 & $76 / \mathrm{M}$ & pT2 & High grade urothelial carcinoma & Neuromyotonia & $\begin{array}{l}\text { Anti- } \\
\text { VGKC }\end{array}$ & Resectionofthetumour & Complete improvement \\
\hline 5 & Sean et al.[14] & 2003 & $59 / \mathrm{M}$ & N/A & N/A & POM & Anti-Ri & N/A & N/A \\
\hline 6 & Charles et al.[22] & 2001 & $57 / M$ & pT3 & High grade urothelial carcinoma & POM & Anti-Ri & $\begin{array}{l}\text { Radical cystectomy and } \\
\text { immunosuppression }\end{array}$ & Partial improvement \\
\hline 7 & Lowe et al.[23] & 1992 & $71 / \mathrm{M}$ & pT3 & High grade urothelial carcinoma & $\begin{array}{l}\text { Visual changes, } \\
\text { glossal spasm and } \\
\text { dysphagia }\end{array}$ & N/A & $\begin{array}{l}\text { Combination } \\
\text { chemotherapy }\end{array}$ & Complete improvement \\
\hline 8 & John et al.[11] & 1999 & $64 / F$ & pT2 & High grade urothelial carcinoma & PCD & Anti-Yo & $\begin{array}{l}\text { Partial resection of the } \\
\text { bladder }\end{array}$ & No improvement \\
\hline 9 & Current report & 2015 & $68 / \mathrm{M}$ & $\mathrm{pCIS}$ & $\begin{array}{l}\text { High grade urothelial carcinoma and the } \\
\text { well-differentiated squamous cell } \\
\text { carcinoma }\end{array}$ & PCD & Negtive & $\begin{array}{l}\text { Laparoscopic radical } \\
\text { cystectomy }\end{array}$ & Complete improvement \\
\hline
\end{tabular}

Paraneoplastic opsoclonus-myoclonus; Anti-CKB anti-creatine kinase, brain-type; N/A Not available 
we believe the PNS is more likely to arise in patients with high grade urothelial carcinoma with squamous differentiation.

Onconeural antibodies are of vital importance in PNS pathogenesis and diagnosis. However, only $60-70 \%$ of patients have detectable onconeural antibodies [10]. Onconeural antibodies were not detected in the present case. Table 3 shows that the bladder cancer antibodies inducing PNS are anti-Ri, anti-Hu, anti-Yo and antiVGKC. SCC and CYFRA21-1 serum concentrations were increased. SCC and CYFRA21-1 are squamous cell carcinoma markers and not paraneoplastic antibodies. No relationship between the low serum thyroglobulin level and PCD is believed to exist. The low serum thyroglobulin level may be due to primary thyroid changes.

Early treatment of the related tumour is the best way to promote neurological improvement. The therapeutic effects of immune therapy were unsatisfactory in these nine cases. The underlying bladder cancer received early diagnosis and timely radical cystectomy. Therefore, injury to the nervous systems was reversible, and PNS disappeared in the present case. Thus, patients with muscle-invasive bladder cancer or CIS of the bladder that simultaneously suffered from PNS require timely radical cystectomy to avoid irreversible injury to the nervous system.

\section{Conclusion}

The patient in the present case suffering from PCD attained a good prognosis. This prognosis was due to early diagnosis and timely radical cystectomy. PNS has an obvious female preponderance in patients with bladder cancers. PNS is more likely to arise in patients with high grade urothelial carcinoma with squamous differentiation. Therefore, timely radical cystectomy is necessary to achieve a good prognosis.

\section{Ethics approval and consent to participate}

The study was approved by the Ethics Committee of Qilu Hospital of Shandong University and the methods were carried out in accordance with the approved guidelines.

\section{Consent for publication}

Written informed consent was obtained from the patient for publication of this case report and any accompanying images. A copy of the written consent is available for review by the Editor of this journal.

\section{Abbreviations}

PNS: paraneoplastic neurological syndromes; PCD: paraneoplastic cerebellar degeneration; TURBt: transurethral resection of bladder tumor;

CIS: Carcinoma in situ; BCG: Bacillus Calmette-Guérin; RC: radical cystectomies; CSF: cerebrospinal fluid; MRI: magnetic resonance imaging; CT: computed tomography.

\section{Competing interests}

The authors declare that they have no competing interest.

\section{Authors' contributions}

YZ and SZC collected the clinical data and drafted the manuscript. SYC and JS revised the manuscript. FC, HG, ZS and YW carried out the clinical management of the patient. CZ carried out the pathological diagnosis and immunohistochemical staining. BS designed the manuscript and tables. All authors have read and approved the manuscript.

\section{Acknowledgements}

We would like to thank the clinical pathologists Prof. Bo Han, Prof. Jianping Zhang and Dr. Wei Gao for kindly helping us with the histopathologic evaluation.

\section{Funding}

This work was supported by the Tai Shan Scholar Foundation to B. Shi, Science Foundation of Qilu Hospital of Shandong University (Grant 2015QLMS28 to B. Shi; Grant 2015QLQN21 to Y. Zhu), Medicine and Health Science Technology Development Project of Shandong Province (Grant 2014 WS0138 to Y. Zhu; Grant 2014WS0144 to C. Zhou).

\section{Author details}

${ }^{1}$ Department of Urology, Qilu Hospital of Shandong University, Wenhua Xi Road, Jinan, Shandong Province, People's Republic of China. ${ }^{2}$ Department of Neurosurgery, Shanghai tenth people's Hospital, Tongji University, Yanchang Zhong Road, Shanghai, People's Republic of China. ${ }^{3}$ Shandong University School of Medicine, Wenhua Xi Road, Jinan, Shandong Province, People's Republic of China.

Received: 15 June 2015 Accepted: 11 May 2016

Published online: 21 May 2016

\section{References}

1. Chavan S, Bray F, Lortet-Tieulent J, Goodman M, Jemal A. International variations in bladder cancer incidence and mortality. Eur Urol. 2014;66(1):59-73.

2. Miyamoto H, Miller JS, Fajardo DA, Lee TK, Netto GJ, Epstein JI. Non-invasive papillary urothelial neoplasms: the 2004 WHO/ISUP classification system. Pathol Int. 2010;60(1):1-8.

3. Richterstetter M, Wullich B, Amann K, Haeberle L, Engehausen DG, Goebell PJ, Krause FS. The value of extended transurethral resection of bladder tumour (TURBT) in the treatment of bladder cancer. BJU Int. 2012;110(2 Pt 2):E76-79.

4. Griffiths TR, Charlton M, Neal DE, Powell PH. Treatment of carcinoma in situ with intravesical bacillus Calmette-Guerin without maintenance. J Urol. 2002;167(6):2408-12

5. Takenaka A, Yamada Y, Miyake H, Hara I, Fujisawa M. Clinical outcomes of bacillus Calmette-Guerin instillation therapy for carcinoma in situ of urinary bladder. Int J Urology. 2008;15(4):309-13.

6. Lamm DL. Carcinoma in situ. Urol Clini North Am. 1992;19(3):499-508.

7. Darnell RB, Posner JB. Paraneoplastic syndromes involving the nervous system. N Engl J Med. 2003;349(16):1543-54.

8. Rosenfeld MR, Dalmau J. Diagnosis and management of paraneoplastic neurologic disorders. Curr Treat Options Oncol. 2013;14(4):528-38.

9. Giometto B, Grisold W, Vitaliani R, Graus F, Honnorat J, Bertolini G, Euronetwork PNS. Paraneoplastic neurologic syndrome in the PNS Euronetwork database: a European study from 20 centers. Arch Neurol. 2010;67(3):330-5.

10. Kannoth S. Paraneoplastic neurologic syndrome: A practical approach. Ann Indian Acad Neurol. 2012;15(1):6-12.

11. Greenlee JE, Dalmau J, Lyons T, Clawson S, Smith RH, Pirch HR. Association of anti-Yo (type I) antibody with paraneoplastic cerebellar degeneration in the setting of transitional cell carcinoma of the bladder: detection of $Y_{O}$ antigen in tumor tissue and fall in antibody titers following tumor removal. Ann Neurol. 1999;45(6):805-9.

12. Tetsuka S, Tominaga K, Ohta E, Kuroiwa K, Sakashita E, Kasashima K, Hamamoto T, Namekawa M, Morita M, Natsui S, et al. Paraneoplastic cerebellar degeneration associated with an onconeural antibody against creatine kinase, brain-type. J Neurol Sci. 2013;335(1-2):48-57.

13. Graus F, Delattre JY, Antoine JC, Dalmau J, Giometto B, Grisold W, Honnorat J, Smitt PS, Vedeler C, Verschuuren JJ, et al. Recommended diagnostic 
criteria for paraneoplastic neurological syndromes. J Neurol Neurosurg Psychiatry. 2004;75(8):1135-40.

14. Pittock SJ, Lucchinetti CF, Lennon VA. Anti-neuronal nuclear autoantibody type 2: paraneoplastic accompaniments. Ann Neurol. 2003;53(5):580-7.

15. Candler PM, Hart PE, Barnett M, Weil R, Rees JH. A follow up study of patients with paraneoplastic neurological disease in the United Kingdom. J Neurol Neurosurg Psychiatry. 2004;75(10):1411-5.

16. Matsumoto L, Yamamoto T, Higashihara M, Sugimoto I, Kowa H, Shibahara J, Nakamura K, Shimizu J, Ugawa Y, Goto J et al. Severe hypokinesis caused by paraneoplastic anti-Ma2 encephalitis associated with bilateral intratubular germ-cell neoplasm of the testes. Mov Disord. 2007;22(5):728-31.

17. Thanarajasingam G, Milone M, Kohli M: Paraneoplastic encephalopathy: an unusual presenting feature of bladder cancer metastasis. BMJ Case Rep. 2015 2015. doi:10.1136/bcr-2014-208913.

18. Dai Y, Li P, Yan S, Xia X, Li Z, Xia M: Lung squamous carcinoma with two paraneoplastic syndromes: dermatomyositis and Lambert-Eaton myasthenic syndrome. Clinical Respir J 2014

19. Bruylant K, Crols R, Humbel RL, Appel B, De Deyn PP. Probably anti-Tr associated paraneoplastic cerebellar degeneration as initial presentation of a squamous cell carcinoma of the lung. Clin Neurol Neurosurg. 2006; 108(4):415-7.

20. Lukacs S, Szabo N, Woodhams S. Rare association of anti-hu antibody positive paraneoplastic neurological syndrome and transitional cell bladder carcinoma. Case Rep Urol. 2012;2012:724940.

21. Forte F, Pretegiani E, Battisti C, Sicurelli F, Federico A. Neuromyotonia as paraneoplastic manifestation of bladder carcinoma. J Neurol Sci. 2009; 280(1-2):111-2.

22. Prestigiacomo CJ, Balmaceda C, Dalmau J. Anti-Ri-associated paraneoplastic opsoclonus-ataxia syndrome in a man with transitional cell carcinoma. Cancer. 2001;91(8):1423-8.

23. Lowe BA, Mershon C, Mangalik A. Paraneoplastic neurological syndrome in transitional cell carcinoma of the bladder. J Urol. 1992;147(2):462-4.

\section{Submit your next manuscript to BioMed Central and we will help you at every step:}

- We accept pre-submission inquiries

- Our selector tool helps you to find the most relevant journal

- We provide round the clock customer support

- Convenient online submission

- Thorough peer review

- Inclusion in PubMed and all major indexing services

- Maximum visibility for your research

Submit your manuscript at www.biomedcentral.com/submit

) Biomed Central 